\title{
Recovery from one-trial overshadowing
}

\author{
ROBERT P. COLE \\ Allied Services, Scranton, Pennsylvania \\ PHILIPPE OBERLING \\ INSERM U405, Strasbourg, France \\ and \\ RALPH R. MILLER \\ State University of New York, Binghamton, New York
}

\begin{abstract}
Prevailing models of associative learning can all account for multitrial overshadowing. However, they fail to account for one-trial overshadowing, which is ordinarily explained in terms of distraction of the subject by the more salient of two simultaneously trained cues from the less salient cue, which interferes with associative acquisition. In the present study, we demonstrate that recovery from overshadowing can be obtained through two techniques that have previously been found to restore responding to overshadowed cues in multitrial training situations. Specifically, recovery from one-trial overshadowing was obtained by extinguishing the overshadowing stimulus and also by administering a posttraining reminder treatment consisting of brief exposure to the overshadowed cue. The similarity of these observations to those in multitrial overshadowing suggests that one-trial and multitrial overshadowing arise from a common underlying mechanism and further augments the view that all cue competition is due (at least in part) to a failure to express acquired information, rather than to a failure to learn.
\end{abstract}

Contemporary theories of associative learning (e.g., Mackintosh, 1975; Pearce \& Hall, 1980; Rescorla \& Wagner, 1972) were all designed largely to explain cue competition. The simplest type of cue competition is overshadowing, in which control of behavior by a target cue $(\mathrm{X})$ is attenuated if it was trained in the presence of another cue (A), as opposed to being trained alone. That is, $\mathrm{AX} \rightarrow$ outcome trials produce less responding to $\mathrm{X}$ than do $X \rightarrow$ outcome trials, particularly if $A$ is more salient (usually meaning more intense) than X. Notably, most contemporary theories of learning predict that overshadowing will occur only after multiple training trials. Owing to the underlying assumptions of attentional (e.g., Mackintosh, 1975; Pearce \& Hall, 1980) and nonattentional (e.g., Rescorla \& Wagner, 1972) models of learning that can account for multitrial overshadowing, neither type of model can account for the occurrence of

Support for this research was provided by National Institute of Mental Health Grant 33881 and by the Foundation Bettencourt-Schueller (France). Philippe Oberling is currently on the faculty at Centre de Neurochimie du CNRS at Strasbourg, France. The authors thank Christy Beneri, Aaron P. Blaisdell, Adam Bristol, Daniel Burger, James C. Denniston, Barry Friedman, Lisa Gunther, David G. Payne, and Hernan I. Savastano for comments on an earlier version of this manuscript. Correspondence concerning this article should be addressed to R. R. Miller, Department of Psychology, State University of New York, Binghamton, NY 13902-6000 (e-mail: rmiller@binghamton.edu). overshadowing when training consists of only one compound trial. These theories all predict that conditioning on the first compound trial of an overshadowing procedure will proceed normally for both the overshadowing cue (A) and the overshadowed cue (X). Thus, for these models, the fact that overshadowing is observed after only a single training trial creates problems (e.g., J. H. James \& Wagner, 1980; Mackintosh \& Reese, 1979).

The failure of all of these theoretical accounts to explain one-trial overshadowing has led to several alternative suggestions. For example, Mackintosh and Reese (1979) suggested that, given a single pairing of a compound of two cues (of differing saliencies) with an outcome, the more salient cue will successfully compete with the less salient cue for a limited amount of perceptual attention. Thus, the perceptual attention allocated to the less salient cue is less than it would have been if that cue had occurred alone. Overshadowing arises because such a decrease in perceptual attention diminishes further processing of the less salient cue on that same trial and, in turn, leads to a deficit in learning. Notably, Mackintosh and Reese contended that competition for perceptual attention and, as a consequence, one-trial overshadowing will occur only if there is an appreciable difference in salience between the two cues.

J. H. James and Wagner (1980) offered an alternative to Mackintosh and Reese's (1979) view. They observed 
one-trial overshadowing when elements of a compound cue were of equal salience and, also, when a serial (i.e., $\mathrm{A} \rightarrow \mathrm{X} \rightarrow$ outcome), rather than a simultaneous (i.e., $\mathrm{AX} \rightarrow$ outcome), overshadowing trial was administered. J. $\mathrm{H}$. James and Wagner argued against the perceptual attention view in light of this demonstration of serial one-trial overshadowing. They doubted whether stimuli not immediately present (A) could engage perceptual attention at the expense of a stimulus that was immediately present (X). J. H. James and Wagner speculated that one-trial overshadowing was the result of postperceptual competition for processing in short-term memory. They posited that elements of a compound cue (regardless of simultaneous or serial presentation) might not gain access to short-term memory processing that is comparable with that gained by individually presented stimuli. In their framework, deficient short-term memory processing of an element of a cue will result in a failure to acquire an association between that deficiently processed cue and the outcome in long-term memory. This explanation foreshadows the account of one-trial overshadowing that flows from Wagner's (1981) SOP model.

Although they differ in detail, both of these accounts of one-trial overshadowing invoked various forms of the concept of divided attention, as developed earlier by Broadbent (1958), W. James (1890), Kahneman (1973), Lovejoy (1968), and Sutherland and Mackintosh (1971), and were unrelated to most contemporary theories of associative learning. Specifically, the more salient cue (A) was assumed to distract the subject from the less salient cue $(\mathrm{X})$, with the result that less is learned about the $\mathrm{X}$ outcome relationship. Wagner's (1981) SOP model of learning does provide a modern formalized version of this distraction account, with one-trial overshadowing arising from competition for space in a limited capacity memory buffer (called Al) between the representations of $A$ and $X$.

An alternative account for one-trial overshadowing is provided by R. R. Miller and Matzel's (1988; see, also, R. R. Miller \& Schachtman, 1985) comparator hypothesis, which is a rule for the expression of Pavlovian associations, rather than for their acquisition. In contrast to Wagner's (1981) SOP model, it views one-trial (as well as multitrial) overshadowing as a failure to express the $\mathrm{X}$-outcome association, rather than as a failure to acquire the $\mathrm{X}$-outcome association. The comparator hypothesis does this by positing that conditioned responding is a direct function, not of a cue's associative value, but of its associative value relative to that of other salient cues that were present during training with the target cue (see Figure 1). That is, subjects presumably respond to the change in the likelihood of the outcome that was signaled by the cue during training, rather than to the absolute likelihood of outcome in the presence of the cue. The present research was designed to differentiate these two views and to gather further evidence bearing on whether one-trial overshadowing is best viewed as being similar to multitrial overshadowing or as the consequence of different underlying mechanisms.

One assumption concerning multitrial overshadowing shared by attentional (e.g., Mackintosh, 1975) as well as nonattentional models (e.g., Rescorla \& Wagner, 1972) is that overshadowing reflects an acquisition deficit, as opposed to a failure to express acquired information. However, this assumption has been challenged. For example, Kaufman and Bolles (1981) and Matzel, Schachtman, and Miller (1985) found that, following AX $\rightarrow$ outcome pairings, many nonreinforced presentations (i.e., extinction) of A facilitated conditioned responding to the overshadowed cue $(\mathrm{X})$. Moreover, posttraining reminder treatments, consisting of brief presentations of the overshadowed cue $(\mathrm{X})$ alone or of the outcome alone, have been found to promote recovery from overshadowing (Kasprow, Cacheiro, Balaz, \& Miller, 1982). These reminder treatments are hypothesized to reactivate a memory trace of the initial training episode, thereby facilitating subsequent retrieval of the association between the overshadowed cue and the outcome on later tests. Under at least select conditions, both of these posttraining treatments (massive extinction of $\mathrm{A}$ and reminder treatments) result in an attenuation of overshadowing without further $\mathrm{X} \rightarrow$ outcome training. These observations of recovery from multitrial overshadowing argue against most accounts of multitrial overshadowing that assume that it is an acquisition deficit (e.g., Mackintosh, 1975; Pearce \& Hall, 1980; Rescorla \& Wagner, 1972; Wagner, 1981), because within these models, only further training with $\mathrm{X}$ should attenuate the overshadowing response deficit (see the Discussion section for a qualification).

Given that traditional acquisition-failure explanations of multitrial overshadowing are challenged by demonstrations of recovery of responding to the overshadowed stimulus, it is reasonable to question the validity of any acquisition-failure account of one-trial overshadowing. If both types of overshadowing prove to be reversible without further training, it would lend support to the view that they arise from a common underlying mechanism, a conclusion that would add a useful constraint for future models of associative learning. Of course, finding that one-trial overshadowing, unlike multitrial overshadowing, is not reversible would also provide a constraint, albeit a different one. Moreover, an investigation of recovery from one-trial overshadowing would assist in differentiating between Wagner's (1981) SOP model and R. R. Miller and Matzel's (1988) comparator hypothesis as explanations of one-trial overshadowing.

Notably, Batsell and Best (1993), Kraemer, Lariviere, and Spear (1988), and J. S. Miller, Jagielo, and Spear (1990) have already presented data demonstrating that one-trial overshadowing of a flavor by an odor wanes with an increasing retention interval. Thus, recovery from onetrial overshadowing has already been demonstrated by means of spontaneous recovery. However, each of these reports had its own focus, and it is important that none 


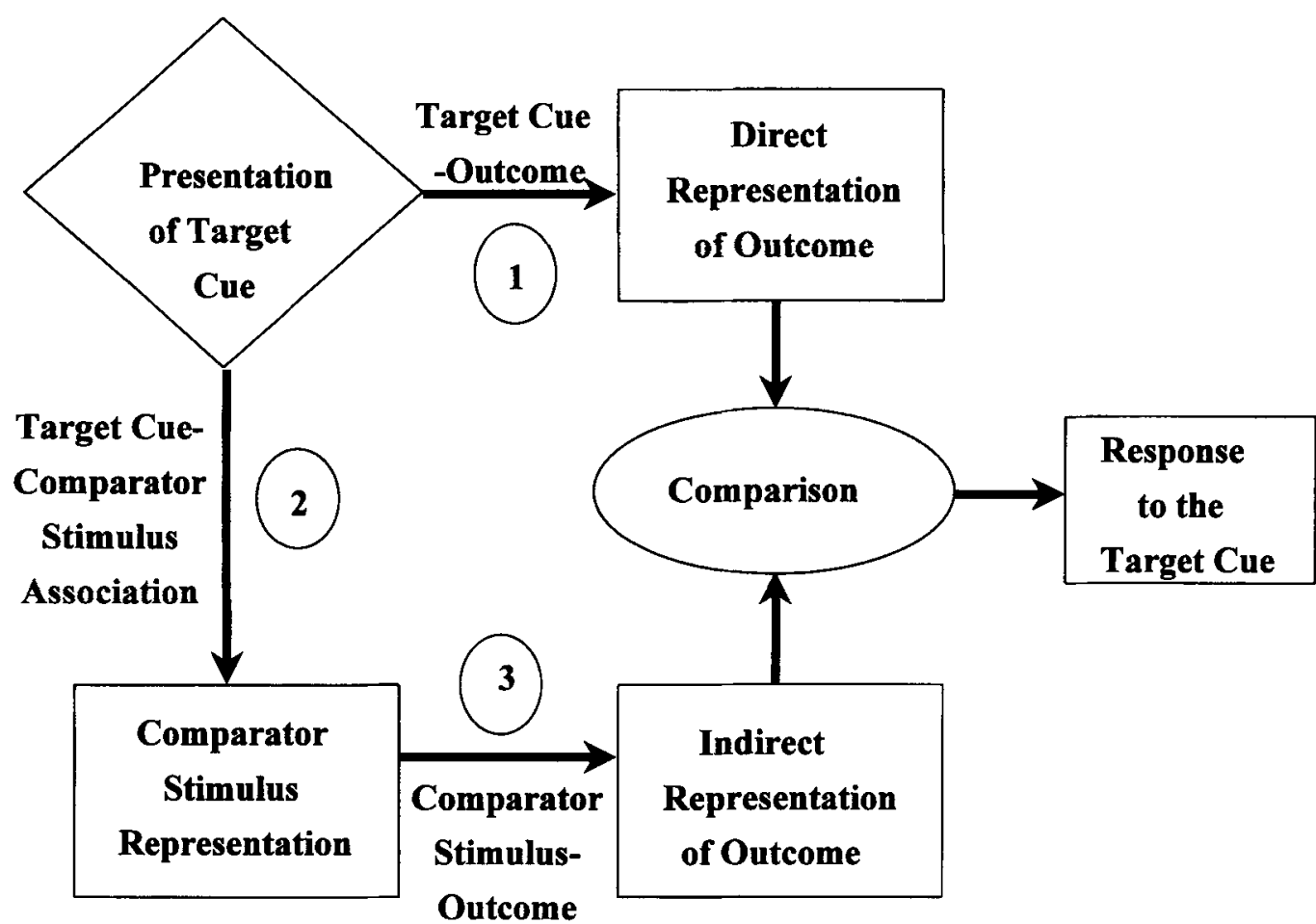

Association

\begin{abstract}
Figure 1: Schematic diagram of the comparator hypothesis, which is a rule for transforming Pavlovian associations into behavior. Presentation of the target cue on the test trial is assumed to activate a direct representation of the outcome as a direct function of the strength of the cue-outcome association (i.e., link 1). The presentation of the test trial cue also is assumed to activate the representation of the most salient other stimulus that was present at the time the test trial cue was trained (i.e., the comparator stimulus), and the degree of activation of the representation of the comparator stimulus is a direct function of the strength of the cue-comparator stimulus association (i.e., link 2). Then, the activated representation of the comparator stimulus, in turn, activates an indirect representation of the outcome in direct relation to the strength of the comparator stimulus-outcome association (i.e., link 3). Finally, strength of responding is determined by a comparison between the degrees of activation of the direct and the indirect representations of the outcome. Responding on the test trial is directly related to the strength of the target cue-outcome association (link 1) and inversely related to the product of the target cuecomparator stimulus association (link 2) and the comparator stimulus-outcome association (link 3). In overshadowing, the overshadowing cue is assumed to serve as the comparator stimulus for the overshadowed cue.
\end{abstract}

of them mentioned the theoretical implications of recovery from one-trial overshadowing, as distinct from multitrial overshadowing. Moreover, all of these reports used taste aversion preparations, which, at least in the past, have sometimes been suggested to follow different rules than do other forms of conditioning (e.g., Garcia, McGowan, \& Green, 1972, who cite the phenomenon of potentiation as evidence in support of this view). Thus, the aim of the present research was (1) to confirm the occurrence of recovery from one-trial overshadowing, using a posttraining manipulation other than spontaneous recovery; (2) to demonstrate recovery, using a preparation other than taste aversion learning; and (3) to draw attention to the theoretical implications of recovery from one-trial overshadowing (in contrast to recovery from multitrial overshadowing). In the present experiment, a conditioned suppression preparation was used to obtain one-trial overshadowing, and recovery from this overshadowing was examined through the administration of massive posttraining extinction of the overshadowing cue and, also, through the administration of posttraining reminder presentations of the overshadowed cue.

\section{METHOD}

\section{Subjects}

The subjects were 24 male $(220-310 \mathrm{~g})$ and 24 female ( $260-$ $385 \mathrm{~g}$ ) experimentally naive Sprague-Dawley rats. The rats were allowed free access to food in their home cages, but access to water was gradually decreased to $10 \mathrm{~min} /$ day prior to the experiment's initiation. The subjects were randomly assigned to one of four groups $(n=12)$, counterbalanced for sex: Group Overshadowing, Group Overshadowing Control, Group Overshadowing- Extinction, and Group Overshadowing-Reminder.

\section{Apparatus}

Twelve experimental chambers, each housed in its own light- and sound-attenuating enclosure, were used. The chambers were of two 
types, differing markedly in shape and construction materials. These chambers served as Context 1 and Context 2, counterbalanced within groups. Each chamber was equipped with a water-filled lick tube that extended into a drinking recess. An infrared photobeam was projected across the recess, $1 \mathrm{~cm}$ in front of the lick tube. In order to drink from the lick tube, the subjects had to insert their heads into the recess, thereby interrupting the photobeam. Thus, the times during which each subject accessed the lick tube were recorded. Four speakers mounted on separate walls of each enclosure could deliver the following auditory stimuli: A 6-sec click train and a white noise (X and Y, counterbalanced), and a tone $(3000 \mathrm{~Hz})$ and a buzzing sound ( $A$ and $B$, counterbalanced). The overshadowing cues ( $A$ and $B$ ) and the overshadowed cues ( $\mathrm{X}$ and $\mathrm{Y}$ ) were presented at 10 and $6 \mathrm{~dB}(\mathrm{C})$, respectively, above ambient background noise $(74 \mathrm{~dB}[\mathrm{C}])$. All the cues were $15 \mathrm{sec}$ in duration, and the outcome (i.e., an unconditioned stimulus, Z) was a $0.5-\mathrm{sec}, 1.0-\mathrm{mA}$ footshock.

\section{Procedure}

The critical aspects of the present experiment are summarized in Table 1. On Day 1, all the subjects were acclimated to Context 1 during a 60 -min session. On Day 2, overshadowing training was administered in Context 1. During a 20 -min session, Groups Overshadowing, Overshadowing-Extinction, and OvershadowingReminder received one reinforced (i.e., followed by Z) AX trial and one reinforced BY trial, whereas Group Overshadowing-Control received one reinforced $X$ trial and one reinforced $Y$ trial. Trial order was counterbalanced within each group. Recovery manipulations were administered on Days 3-7 outside of the context used for training and testing. The shift in context was done to prevent possible new learning of $\mathrm{A}$-context or $\mathrm{X}$-context associations that would have confounded interpretation of any observed recovery effect. Possibly, the contextual specificity of extinction of A (renewal) and reminder effects worked against the efficacy of our recovery treatments, but we felt it important to preclude interpretations of any observed recovery on the basis of new associations to the training/ test context. Group Overshadowing-Extinction received 36 nonreinforced (i.e., extinction) exposures daily to the overshadowing cue (A) in Context 2, pseudorandomly distributed during 120-min sessions. On Day 7, Group Overshadowing-Reminder received a 10min reminder session in Context 2, which consisted of two 10-sec nonreinforced presentations of X. Groups Overshadowing and Overshadowing-Control remained in their home cages during these sessions but received equivalent handling. Recovery manipulations were conducted in a context other than the training context, because we did not want the various groups to have differential exposure to the training/test context; posttraining exposure to training context alone has sometimes been observed to weaken cue competition (see, e.g., Balaz, Gutsin, Cacheiro, \& Miller, 1982). Other research in our laboratory (Gunther, Denniston, \& Miller, 1998) has suggested that extinction of an overshadowing stimulus (in multitrial situations) is more effective in reversing overshadowing when it occurs in the training context, rather than in a neutral context, but we avoided the training context for our recovery manipulations in the present research because using it would admit a greater number of interpretations of any observed recovery from overshadowing. On Days 8 and 9 , daily 30 -min reacclimation sessions similar to those administered on Day 1 were administered in Context 1 . Reacclimation served to restabilize baseline licking, the departure from which served as the dependent variable during testing.

Testing was conducted over two sessions. On Day 10, all the animals were tested in Context 1 for suppression of ongoing licking behavior in the presence of either X or Y, counterbalanced within groups. During the test session, the animals were allowed to drink from the lick tubes for 5 cumulative seconds, at which time the test stimulus was presented and remained on for $10 \mathrm{~min}$. Thus, all the subjects were drinking at cue onset. Times to complete an additional 5 cumulative seconds of licking in the presence of the test stimulus were recorded. On Day 11, a second test was administered, during which the subjects tested with $\mathrm{X}$ on Day 10 were tested with $\mathrm{Y}$, and vice versa.

Prior to statistical analysis, all the suppression data were converted to $\log$ (base 10) scores, to better meet the assumptions of parametric analysis. The critical aspects of the experiment are summarized in Table 1. Prior to testing, 1 animal from Group Overshadowing became ill and was consequently eliminated from the experiment. A .05 alpha level was adopted for all statistical tests.

\section{RESULTS}

On neither test day were there any significant differences between groups in times from placement in the test chambers to completion of 5 cumulative seconds of drinking (i.e., immediately prior to CS onset; $p s>.20$ ); thus, baseline drinking rates were highly similar across groups.

Suppression means are illustrated in Figure 2. Stimuli $\mathrm{X}$ and $\mathrm{Y}$ were treated identically within Group Over-

Table 1

Experimental Design

\begin{tabular}{|c|c|c|c|}
\hline Group & $\begin{array}{l}\text { Overshadowing } \\
\text { Training }\end{array}$ & $\begin{array}{c}\text { Posttraining } \\
\text { Recovery Treatment }\end{array}$ & Test \\
\hline $\begin{array}{l}\text { Overshadowing } \\
\text { Overshadowing } \\
\text { Control }\end{array}$ & $\begin{array}{l}{[\mathrm{AX} \rightarrow \mathrm{Z}]_{1} /[\mathrm{BY} \rightarrow \mathrm{Z}]_{1}} \\
{[\mathrm{X} \rightarrow \mathrm{Z}]_{1} /[\mathrm{Y} \rightarrow \mathrm{Z}]_{1}}\end{array}$ & & $\begin{array}{l}{[\mathrm{X}]_{1} \text { and }[\mathrm{Y}]_{1}} \\
{[\mathrm{X}]_{1} \text { and }[\mathrm{Y}]_{1}}\end{array}$ \\
\hline & {$[\mathrm{AX} \rightarrow \mathrm{Z}]_{\mathrm{l}} /[\mathrm{BY} \rightarrow \mathrm{Z}]_{\mathrm{l}}$} & {$[\mathrm{A}-]_{2}$} & {$[\mathrm{X}]_{1}$ and $[\mathrm{Y}]_{1}$} \\
\hline $\begin{array}{l}\text { Overshadowing- } \\
\text { Reminder }\end{array}$ & {$[\mathrm{AX} \rightarrow \mathrm{Z}]_{1} /[\mathrm{BY} \rightarrow \mathrm{Z}]_{1}$} & {$[\mathrm{X}-]_{2}$} & {$[\mathrm{X}]_{1}$ and $[\mathrm{Y}]_{1}$} \\
\hline \multicolumn{4}{|c|}{ 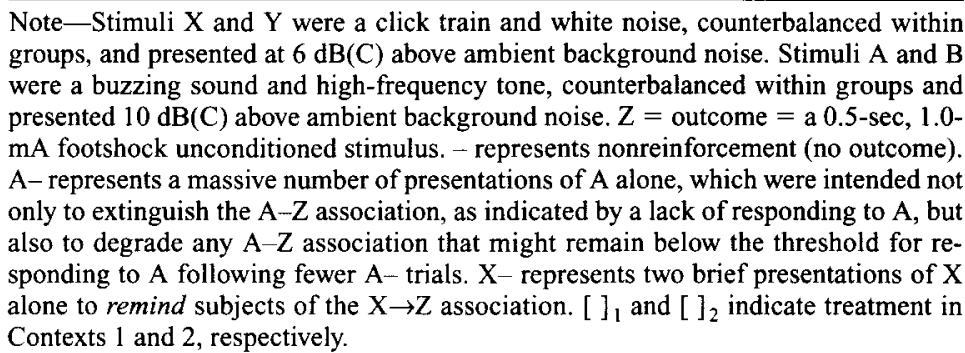 } \\
\hline
\end{tabular}




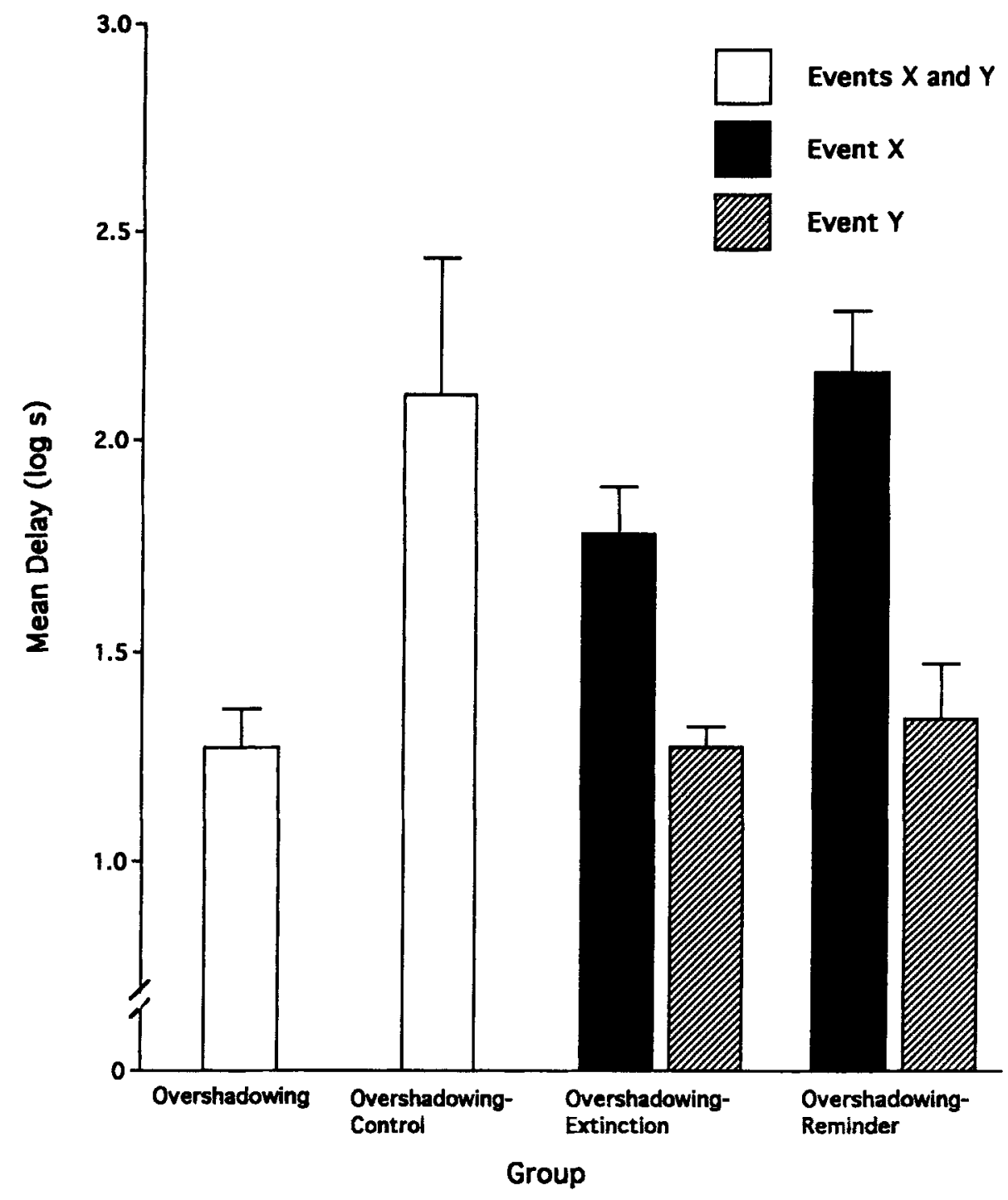

Figure 2. Solid bars represent mean delay in log seconds to drink for 5 cumulative seconds in the presence of the overshadowed cue, $X$. Hatched bars represent mean delay in log seconds to drink for 5 cumulative seconds in the presence of the overshadowed cue, $Y$. The open bars represent the pooled $X$ and $Y$ data in Groups Overshadowing and Overshadowing-Control, for which $X$ and $Y$ were treated identically and counterbalanced as to physical stimulus. Error bars denote the standard error of the mean.

shadowing and again within Group OvershadowingControl. Thus, any attempt at distinguishing them within these groups would have been arbitrary. Consequently, $\mathrm{X}$ and $\mathrm{Y}$ scores within subjects were pooled. $\mathrm{A} t$ test between these two groups revealed a weaker response in Group Overshadowing $[t(21)=2.54]$, which is indicative of A and B's having overshadowed $\mathrm{X}$ and $\mathrm{Y}$.

For purposes of further analysis, scores from Groups Overshadowing and Overshadowing-Control were arbitrarily designated $\mathrm{X}$ or $\mathrm{Y}$, counterbalanced for subject, physical stimulus, and test day. This permitted us to conduct a 2 (group, overshadowing vs. overshadowingextinction) $\times 2$ (test stimulus, $X$ vs. $Y$ ) analysis of vari- ance (ANOVA), which revealed main effects of group $[F(1,21)=9.14]$ and test stimulus $[F(1,21)=6.45]$ and also a group $\times$ test stimulus interaction $[F(1,21)=4.64]$. As can be seen in Figure 2, the source of this interaction arose from greater suppression to $\mathrm{X}$ than to $\mathrm{Y}$ by Group Overshadowing-Extinction, relative to Group Overshadowing. This interaction indicates that extensive extinction of the overshadowing stimulus $\mathrm{A}$ in a novel context increased excitatory control by $\mathrm{X}$ - that is, Group Overshadowing-Extinction responded more to $X$ than did Group Overshadowing $[F(1,21)=11.13]$ —but did not increase excitatory control by $\mathrm{Y}$ - that is, responding to $\mathrm{Y}$ did not differ between Group Overshadowing-Ex- 
tinction and Group Overshadowing $[F(1,21)=0.07]$. This increase in the excitatory control by $\mathrm{X}$ in Group Overshadowing-Extinction demonstrates that one-trial overshadowing can be attenuated without further $X \rightarrow Z$ training. In addition, the failure to observe a similar increase of excitatory control by $\mathrm{Y}$ in Group Overshadowing-Extinction indicates that this recovery effect is stimulus specific (i.e., extinction of an overshadowing cue [A] exclusively enhanced responding to the overshadowed cue $[\mathrm{X}]$ with which it was paired during training).

A second, analogous $2 \times 2$ ANOVA compared responding to $\mathrm{X}$ and $\mathrm{Y}$ in Groups Overshadowing and Overshadowing-Reminder. This analysis revealed a main effect of group $[F(1,21)=10.19]$, a main effect of test stimulus $[F(1,21)=18.09]$, and a group $\times$ test stimulus interaction $[F(1,21)=14.42]$. Inspection of Figure 2 suggests that the source of this interaction is the relatively greater amount of suppression to $X$ than to $Y$ by Group Overshadowing-Reminder, relative to Group Overshadowing. This finding indicates that a posttraining reminder treatment consisting of brief nonreinforced presentations of an overshadowed stimulus in a novel context can attenuate the one-trial overshadowing effect - that is, nonreinforced presentations of the overshadowed cue $(X)$ led to facilitated responding to $\mathrm{X}$ by Group OvershadowingReminder, relative to Group Overshadowing $[F(1,21)=$ 47.20]. Moreover, the failure of the $X$ reminder treatment to facilitate conditioned responding to $Y$ in Group Overshadowing-Reminder, relative to responding to $Y$ in Group Overshadowing $[F(1,21)=2.36]$, indicates that this reminder-induced recovery from one-trial overshadowing is stimulus specific (i.e., administration of a target cue reminder treatment exclusively enhanced responding to that overshadowed cue).

Thus, the present data replicated the one-trial overshadowing effect with a simultaneous compound cue consisting of two elements of differing intensities. Also, recovery from the one-trial overshadowing effect was obtained as a result of posttraining extinction of the overshadowing cue. In addition, a reminder treatment consisting of two nonreinforced presentations of an overshadowed cue was effective in attenuating the one-trial overshadowing effect. In both cases, recovery was specific to the overshadowed cue for which the recovery treatment had been given.

\section{DISCUSSION}

The present experiment illuminates the nature of onetrial overshadowing. We confirmed the occurrence of recovery from one-trial overshadowing with two posttraining manipulations, both different from the spontaneous recovery design that had been used successfully, but without attention to the implications of a one-trial training procedure (Batsell \& Best, 1993; Kraemer, Lariviere, \& Spear, 1988; J. S. Miller, Jagielo, \& Spear, 1990). Moreover, we demonstrated this recovery with a conditioned suppression preparation, in contrast with earlier work, which had consistently used taste aversion learning. Below, we discuss the theoretical implications of these observations.

The explanations that were originally advanced to account for one-trial overshadowing (J. H. James \& Wagner, 1980; Mackintosh \& Reese, 1979) suggested that the one-trial overshadowing deficit is due to the competing cues' conjointly exceeding the capacity of some perceptual or short-term memory attentional resource, thereby precluding further processing of (and learning about) the overshadowed stimulus. The present and past demonstrations of recovery from the one-trial overshadowing deficit indicate that these earlier accounts are inadequate. Note that recovery from one-trial overshadowing was obtained without additional pairings of the overshadowed antecedent cue and the outcome following overshadowing training. This suggests that the response deficit seen in one-trial overshadowing, at least with our preparation, is due to a failure to behaviorally express acquired information, rather than to a failure to acquire the information.

Researchers (e.g., J. H. James \& Wagner, 1980; Mackintosh \& Reese, 1979) initially proposed that one-trial overshadowing is governed by an underlying process different from that governing multitrial overshadowing, because prevailing accounts of multitrial overshadowing were unable to account for one-trial overshadowing. However, the previously cited reports that multitrial overshadowing is, at least in part, due to a failure to express information that was acquired indicates that these prevailing accounts of multitrial overshadowing (acquisition-focused models, such as Mackintosh, 1975; Pearce \& Hall, 1980; Rescorla \& Wagner, 1972) are as unable to account for multitrial overshadowing as they are to account for onetrial overshadowing. The only contemporary theory of learning that could account for one-trial overshadowing per se was Wagner's (1981) SOP model. However, SOP cannot account for posttraining recovery from one-trial overshadowing.

An alternative explanation of recovery from overshadowing as a consequence of extinguishing the overshadowing cue is provided by Van Hamme and Wasserman (1994) and Dickinson and Burke (1996). These investigators have proposed models of associative learning that view overshadowing as an acquisition deficit and explain the observed recovery from multitrial overshadowing that results from posttraining extinction of the overshadowing cue as a consequence of further strengthening of the $\mathrm{X}$-outcome association on the A extinction trials, despite the absence of X on these trials. But Van Hamme and Wasserman (a variant of the Rescorla-Wagner model) cannot explain the occurrence of one-trial overshadowing itself, for the same reason that Rescorla and Wagner (1972) cannot, whereas Dickinson and Burke (a variant of Wagner's [1981] SOP model) can, by the same mechanism as 
that for SOP. However, recovery from one-trial (or multitrial) overshadowing effected by a cue-reminder treatment is diametrically opposed to expectations based on Dickinson and Burke's version of SOP, which anticipates that the overshadowed cue should accrue inhibitory value (if anything) as a consequence of nonreinforced posttraining exposures alone. That is, according to Dickinson and Burke, presentation of the overshadowed cue on a reminder trial should have activated a representation of the cue in memory state $A 1$ and, through retrieval, activated a representation of the outcome in memory state A2. According to Wagner and to Dickinson and Burke, having a cue representation in $\mathrm{A} 1$ and an outcome in $\mathrm{A} 2$ at the same time are the conditions that automatically create an inhibitory association from the cue to the outcome; that is, the overshadowed cue should become inhibitory with respect to the outcome, not more excitatory.

Notably, both one-trial and multitrial overshadowing appear to be reversible, thereby encouraging the view that they arise from a common underlying process. The comparator hypothesis (R. R. Miller \& Matzel, 1988) offers an explanation of one-trial overshadowing that is equally applicable to multitrial overshadowing. Specifically, during overshadowing treatment of $\mathrm{AX} \rightarrow$ outcome, an $\mathrm{X}-\mathrm{A}$ association and an $\mathrm{A}-$ outcome association are formed, so that, at test, $X$ not only activates a direct representation of the outcome through the $\mathrm{X}$-outcome association, but also a strong indirect representation of the outcome through the conjoint action of the $\mathrm{X}-\mathrm{A}$ and the $\mathrm{A}$-outcome associations. Conditioned responding to $X$ is assumed to be inversely related to the magnitude of the activated indirect outcome representation; hence, both one-trial and multitrial overshadowing are anticipated. Moreover, the comparator hypothesis correctly predicts that posttraining extinction of the overshadowing cue (A) will restore responding to the overshadowed cue in both the one-trial case (i.e., the present data) and the multitrial case (e.g., Dickinson \& Charnock, 1985; Kaufman \& Bolles, 1981; Matzel et al., 1985; but see Rescorla \& Cunningham, 1978). Presumably, posttraining extinction of A degraded the $A \rightarrow Z$ association (i.e., link 3 in Figure 1) and, possibly, the $X \rightarrow A$ association (i.e., link 2 in Figure 1), thereby reducing the magnitude of the indirect representation of the outcome $(Z)$ that was activated by $X$ on the test trial. The comparator hypothesis is less readily able to explain how reminder treatments work to restore responding to an overshadowed cue. However, the reversibility of overshadowing that is seen as a consequence of reminder treatment is at least consistent with the spirit of the comparator hypothesis, which posits that contiguity alone is sufficient for associative acquisition and that cue competition deficits in conditioned responding (including overshadowing) are failures to express associations that were acquired. Specific mechanisms that might account for the effect of the reminder treatment would include perceptual learning about X, making $X$ more salient so that it would, at test, be more effective at retrieving the $\mathrm{X} \rightarrow \mathrm{Z}$ association.

Thus, at this time, the comparator hypothesis of R. R. Miller and Matzel (1988) appears to be the model most compatible with the present observations, despite its lack of a specific mechanism to explain the present reminder treatment data. More generally, the present data suggest that one-trial overshadowing and multitrial overshadowing are both reversible and that is is likely that they arise from the same underlying processes.

\section{REFERENCES}

Balaz, M. A., Gutsin, P., Cacheiro, H., \& Miller, R. R. (1982). Blocking as a retrieval failure: Reactivation of associations to a blocked stimulus. Quarterly Journal of Experimental Psychology, 34B, 99-113.

Batsell, W. R., JR., \& Best, M. R. (1993). One bottle too many? Method of testing determines the detection of overshadowing and retention of taste aversions. Animal Learning \& Behavior, 21, 154-158.

Broadbent, D. E. (1958). Perception and communication. London: Pergamon.

Dickinson, A., \& Burke, J. (1996). Within-compound associations mediate the retrospective revaluation of causality judgements. Quarterly Journal of Experimental Psychology, 49B, 60-80.

Dickinson, A., \& CharnoCK, D. J. (1985). Contingency effects with maintained instrumental reinforcement. Quarterly Journal of Experimental Psychology, 37B, 397-416.

Garcia, J., McGowan, B. K., \& Green, K. F. (1972). Biological constraints on conditioning. In A. H. Black \& W. F. Prokasy (Eds.), Classical conditioning II: Current theory and research. New York: Appleton-Century-Crofts.

Gunther, L. M., Denniston, J. C., \& Miller, R. R. (1998). Renewal of comparator stimuli. Learning \& Motivation, 29, 200-219.

JAMES, J. H., \& WAGNER, A. R. (1980). One-trial overshadowing: Evidence of distributive processing. Journal of Experimental Psychology: Animal Behavior Processes, 6, 188-205.

JAMES, W. (1890). Principles of psychology (Vol. 1). New York: Holt.

Kahneman, D. (1973). Attention and effort. Englewood Cliffs, NJ: Prentice-Hall.

Kasprow, W. J., Cacheiro, H., Balaz, M. A., \& Miller, R. R. (1982). Reminder-induced recovery of associations to an overshadowed stimulus. Learning \& Motivation, 13, 155-166.

Kaufman, M. A., \& Bolles, R. C. (1981). A nonassociative aspect of overshadowing. Bulletin of the Psychonomic Society, 18, 318-320.

Kraemer, P. J., Lariviere, N. A., \& SPEAR, N. E. (1988). Expression of a taste aversion conditioned with an odor-taste compound: Overshadowing is relatively weak in weanlings and decreases over a retention interval in adults. Animal Learning \& Behavior, 16, 164-168.

LoveJoy, E. (1968). Attention in discrimination learning. San Francisco: Holden-Day.

Mackintosh, N. J. (1975). A theory of selective attention: Variations in the associability of stimuli with reinforcement. Psychological Review, 82, 276-298.

Mackintosh, N. J., \& Reese, B. (1979). One-trial overshadowing. Quarterly Journal of Experimental Psychology, 31, 519.526.

Matzel, L. D., Schachtman, T. R., \& Miller, R. R. (1985). Recovery of an overshadowed association achieved by extinction of the overshadowing stimulus. Learning \& Motivation, 16, 398-412.

Miller, J. S., Jagielo, J. A., \& Spear, N. E. (1990). Changes in the retrievability of associations to elements of the compound CS determine the expression of overshadowing. Animal Learning \& Behavior, 18, $157-161$.

Miller, R. R., \& MATzel, L. D. (1988). The comparator hypothesis: A response rule for the expression of associations. In G. H. Bower (Ed.), The psychology of learning and motivation (Vol. 22, pp. 5192). San Diego: Academic Press. 
Miller, R. R., \& Schachtman, T. R. (1985). Conditioning context as an associative baseline: Implications for response generation and the nature of conditioned inhibition. In R. R. Miller \& N. E. Spear (Eds.), Information processing in animals: Conditioned inhibition (pp. 51 88). Hillsdale, NJ: Erlbaum

Pearce, J. M., \& H HLL, G. (1980). A model for Pavlovian learning Variations in the effectiveness of conditioned but not of unconditioned stimuli. Psychological Review, 87, 532-552.

Rescorla, R. A., \& Cunningham, C. L. (1978). Within-compound flavor associations. Journal of Experimental Psychology: Animal Be havior Processes, 4, 267-275.

RESCORLA, R. A., \& WAGNER, A. R. (1972). A theory of Pavlovian conditioning: Variations in the effectiveness of reinforcement and non reinforcement. In A. H. Black \& W. F. Prokasy (Eds.), Classical con- ditioning II: Current research and theory (pp. 64-99). New York: Appleton-Century-Crofts.

Sutherland, N. S., \& Mackintosh, N. J. (1971). Mechanisms of animal discrimination learning. New York: Academic Press.

Van Hamme, L. J., \& Wasserman, E. A. (1994). Cue competition in causality judgments: The role of nonpresentation of compound stimulus elements. Learning \& Motivation, 25, 127-151.

WAGNER, A. R. (1981). SOP: A model of automatic memory processing in animal behavior. In N. E. Spear \& R. R. Miller (Eds.), Information processing in animals: Memory mechanisms (pp. 5-47). Hillsdale, NJ: Erlbaum.

(Manuscript received July 13, 1998;

revision accepted for publication November 12, 1998.) 